\title{
EXPERIMENTAL DETERMINATION OF LINEAR OPTICS INCLUDING QUADRUPOLE ROTATIONS*
}

\author{
J. Safranek \\ National Synchrotron Light Source, Brookhaven National Laboratory, Upton, NY 11973
}

\begin{abstract}
The measured response matrix giving the change in orbit at beam position monitors (BPMs) with changes in steering magnet excitation can be used to accurately determine many important parameters in a storage ring. Using the NSLS X-Ray Ring measured response matrix we have determined the gradients in all 56 quadrupole magnets; the calibration of the steering magnets and BPMs; the rotational mis-alignments of the quadrupoles, steering magnets, and BPMs about the electron beam direction; the longitudinal magnetic centers of the orbit steering magnets; and the transverse mis-alignments of the sextupoles. Random orbit measurement error of the BPMs propagated to give $0.04 \% \mathrm{rms}$ error in determination of individual quadrupole gradients and $0.4 \mathrm{mrad} \mathrm{rms}$ error in the determination of quadrupole rotational alignment. Small variations of a few parts in a thousand in the quadrupole gradients within an individual family were resolved. The improved understanding of the X-Ray Ring has enabled us to better control the electron beam size.
\end{abstract}

\section{INTRODUCTION}

A precise understanding of the linear optics, including coupling, in a storage ring is critical to achieving maximum performance. Recent results from Fermilab [1] illustrate the importance of understanding and controlling coupling in colliding beam machines. Careful coupling control will also be crucial in damping rings for linear colliders. In synchrotron light sources, minimization of coupling minimizes the vertical electron beam size and produces the brightest possible photon beam. Here we will present a method for experimentally determining the sources of coupling in a storage ring.

Previous work [2-7] has shown that it is possible to accurately derive the normal gradient distribution in a storage ring by analyzing the orbit response matrix. In this paper this technique will be extended to include a derivation of the skew gradient distribution in the NSLS X-Ray Ring. The X-Ray Ring BPM system permits fast, highly accurate measurement of the orbit response matrix [8]. In less than one second, the orbit at all 48 horizontal and vertical BPMs is read 256 times and averaged. The result is a reading of the orbit limited mostly by the $2.5 \mu \mathrm{m}$ digital resolution. These accurate orbit response measurements yield detailed information concerning the X-Ray Ring optics.

\footnotetext{
*Work performed under the auspices of the U.S. Department of Energy
}

II. METHOD

The MAD [9] accelerator optics modeling program was used to calculate the model response matrix. The parameters in the MAD model were varied to minimize the $\chi^{2}$ deviation between the model and measured orbit response matrices $\left(M_{\text {mod }}\right.$ and $\left.M_{\text {meas }}\right)$.

$$
\chi^{2}=\sum_{i, j} \frac{\left(M_{m e a s, i j}-M_{m o d, i j}\right)^{2}}{\sigma_{i}^{2}}
$$

where the sum is over the 90 orbit steering magnets (51 horizontal and 39 vertical) and the 96 BPMs (48 horizontal and 48 vertical). The matrices include the coupling terms (i.e. the shift in vertical orbit with horizontal steering magnets and horizontal orbit shifts with vertical steering). The $\sigma_{i}$ are the measured noise levels for the BPMs. The $\chi^{2}$ minimization was achieved by iteratively solving the linear system of equations,

$$
-V_{k}=\frac{d V_{k}}{d x_{n}} \Delta x_{n}
$$

where $V_{k}=\left(M_{\text {meas }, i j}-M_{\text {mod, } i j}\right) / \sigma_{i}$ with $k$ ranging from 1 to 8640 for the 8640 elements of the orbit response matrix. The $x_{n}$ are the parameters varied to fit $M_{\text {meas }}$ to $M_{\text {mod }}$. Solving equation 1 for $\Delta x_{n}$ gives the change in the parameters to minimize $\sum_{k} V_{k}^{2}$ which is equivalent to $\chi^{2}$.

The parameters varied to fit $M_{\text {meas }}$ to $M_{\text {mod }}$ include each of the gradients in the $56 \mathrm{X}$-Ray Ring quadrupoles; the small gradient in the dipole magnets; the gains of the 96 BPMs; the calibrations of the 90 orbit steering magnets; and the rotational alignment of the quadrupoles, steering magnets, and BPMs. Also included in the fit is the energy shift associated with changing each orbit steering magnet. When a steering magnet strength is changed, the total path length around the ring must stay constant to keep the electron bunches in synchronism with the rf, so there is an energy shift of the stored beam with an associated shift in the closed orbit proportional to the dispersion.

A fourth parameter was varied for each BPM. Three parameters for each BPM were already mentioned: the horizontal gain, the vertical gain, and the rotational alignment. By adding a fourth parameter we are varying all the possible parameters of a two-dimensional linear fit between the two signals from the BPM and the actual horizontal and vertical orbit. This is necessary, because there is significant variation of the linear mapping from BPM to BPM. The BPMs in the X-Ray Ring were constructed by welding a disk with two pick-up electrodes to the top of the vacuum chamber and another such disk to the bottom of the vacuum chamber. Due to the tolerances in this welding 
process, there is significant variation in the response of the BPMs. The following gives the full linear transformation used in fitting the orbit measurements for each BPM:

$$
\left(\begin{array}{l}
\bar{x} \\
\bar{y}
\end{array}\right)=\frac{1}{\sqrt{1-C^{2}}}\left(\begin{array}{cc}
\cos \theta & \sin \theta \\
-\sin \theta & \cos \theta
\end{array}\right)\left(\begin{array}{ll}
1 & C \\
C & 1
\end{array}\right)\left(\begin{array}{l}
g_{x} x \\
g_{y} y
\end{array}\right) .
$$

The four parameters varied for each BPM are horizontal gain $\left(g_{x}\right)$, vertical gain $\left(g_{y}\right)$, rotation $(\theta)$, and $C$ which is a parameter associated with errors in the construction of the BPM in which one diagonal pair of pick-up electrodes is closer together than the other diagonal pair.

Even with the above parameters varied, the orbit response of certain orbit steering magnets could not be fit to the BPM measurement noise level. The magnets with the poorest fit were the ones closest to other ferromagnetic material in the ring. The steering magnets have long endfields. When they are located near other ferromagnetic material, the end fields are clipped and the longitudinal magnetic center of the steering magnet is shifted from its physical center. We varied the positions of the steering magnets in the MAD model, so the fitting converged to give the longitudinal magnetic centers of the magnets. As expected, we found that the closer a steering magnet was to some other ferromagnetic ring element, the more the fit for its magnetic center deviated from its measured physical center. With the corrected longitudinal position, the model and measured responses agreed to about the noise level of the BPMs.

In all, 626 parameters were varied to fit the 8640 elements in the X-Ray Ring response matrix. When the fit had converged, the rms difference between $M_{\text {mod }}$ and $M_{\text {meas }}$ was $1.2 \mu \mathrm{m}$ which is primarily due to the digital accuracy (one bit is $2.5 \mu \mathrm{m}$ ) of the BPM readings. The fit converged to values to each of the 626 parameters. In the next sections we discuss how accurately these parameters reflect the real gradients, rotations, and calibrations of the elements in the X-Ray Ring.

\section{ERROR ANALYSIS}

Random errors on measured data, such as the random noise on the orbit response matrix measurement, propogate in a predictable way to give well-defined error bars on fit parameters. Unknown systematic errors, on the other hand, propogate in unknown ways, making it is difficult to determine the size of the error bars. Every effort was made to ensure that the difference between the model and measured response matrices converged to the noise level of the BPMs, because this ensures that there is no remaining systematic error in the model. If all 626 parameters were not included in the fit, the rms difference between the model and measured response matrices would not have converged to $1.2 \mu \mathrm{m}$. The additional error would have been due to systematic error and would have contributed an unknown amount to the error bars.

The number of data points, 8640 , is much greater than the number of parameters, 626 , but this does not in itself guarantee that the solution is unique. One way to test for uniqueness is to look at the eigenvalues associated with the matrix, $d V_{k} / d x_{n}$, in equation 1 . If this matrix is singular, it will have eigenvalue(s) equal to zero, and there will be an infinite region in parameter space over which the fit gives the minimum $\chi^{2}$. In such a case, our fit parameters would have infinite error bars regardless of how small the BPM noise is. There is actually one singularity in $d V_{k} / d x_{n}$, which is due to the fact that if all the steering magnet calibrations and all the BPM gains were increased together, the response matrix would not change. This means that when analyzing the orbit response matrix data alone, only the relative calibrations of the BPMs and steering magnets can be derived. The absolute calibrations have infinite error bars. The absolute calibrations, however, can be derived by comparing the shift in orbit with $\mathrm{rf}$ frequency to the model dispersion. The model dispersion is well known from the quadrupole calibration, so the absolute gain calibration of the BPMs can be derived. The singularity in $d V_{k} / d x_{n}$ was avoided by inverting the matrix using singular value decomposition (SVD) [10]. The SVD threshold was adjusted to eliminate the one very small eigenvalue. The eigenvalue associated with the steering magnet/BPM degeneracy was 15 times smaller than the next smallest eigenvalue. That all the other eigenvalues were much larger indicates that there are no other degeneracies in $d V_{k} / d x_{n}$ (see [10]).

The easiest way to determine how much the fit parameters vary due to random errors in the measurements is simply to take many data sets, analyze each one separately, and see how much variation there is between fit parameters for the different data sets. We measured the response matrix ten times, and fit a model to each response matrix. Then, for each of the parameters we took the average over the ten data sets and calculated the rms variation from the average. The results are shown in table 1 .

Table 1. These rms variations are the error bars on the fit parameters due to random orbit measurement errors.

\begin{tabular}{|c|c|}
\hline Parameter & rms variation \\
\hline quadrupole gradients & $.04 \%$ \\
quadrupole rotations & $.4 \mathrm{mrad}$ \\
BPM gain & $.05 \%$ \\
BPM rotations & $.5 \mathrm{mrad}$ \\
BPM C-parameter & .0004 \\
steering magnet calibration & $.05 \%$ \\
steering magnet rotations & $.8 \mathrm{mrad}$ \\
steering magnet longitudinal center & $2 \mathrm{~mm}$ \\
steering magnet fractional energy shift & $3.4 \mathrm{E}-7$ \\
\hline
\end{tabular}

The size of the error bars in table 1 is determined by the signal-to-noise ratio of the orbit response matrix measurement. To decrease the error bars, the signal-to-noise must be increased. The size of the signal is the size of the orbit shifts when measuring the response matrix. Orbit shifts of $.8 \mathrm{~mm}$ were used when measuring the 10 response matrices used for table 1 . We also measured response matrices with $1.6 \mathrm{~mm}$ rms orbit shifts to double the signal 


\section{DISCLAIMER}

This report was prepared as an account of work sponsored by an agency of the United States Government. Neither the United States Government nor any agency thereof, nor any of their employees, makes any warranty, express or implied, or assumes any legal liability or responsibility for the accuracy, completeness, or usefulness of any information, apparatus, product, or process disclosed, or represents that its use would not infringe privately owned rights. Reference herein to any specific commercial product, process, or service by trade name, trademark, manufacturer, or otherwise does not necessarily constitute or imply its endorsement, recommendation, or favoring by the United States Government or any agency thereof. The views and opinions of authors expressed herein do not necessarily state or reflect those of the United States Government or any agency thereof. 


\section{DISCLAIMER}

Portions of this document may be illegible in electronic image products. Images are produced from the best available original document. 
to noise. With $1.6 \mathrm{~mm}$ rms orbit distortions, however, we could only fit $M_{\text {mod }}$ to $M_{\text {meas }}$ to an rms difference of 1.4 $\mu \mathrm{m}$, not the $1.2 \mu \mathrm{m}$ with which we could fit the $.8 \mathrm{~mm}$ rms orbit distortions. This means there were systematic errors in the measurement, most likely due to nonlinearities in the BPM electronics. Thus BPM-electronics nonlinearities limit the size of the signal we can fit. Improved BPM electronics have been developed [11], and will be available for orbit measurements in the future. The present limit on the noise of the orbit response matrix measurement is the 2.5 $\mu m$ digital resolution.

\section{RESULTS}

The error analysis showed that the fit parameters are very close to the real parameters in the X-Ray Ring. As discussed previously [3], the fit quadrupole gradients agreed well with the magnetic measurements we were able to find. Also other measured lattice parameters such as dispersion and tunes agreed well with the MAD model the fitting generated.

Table 2 shows the rms size of the fit rotations as well as the maximum rotations found. The BPM rotations are quite large due to the construction method described above.

Table 2. This table shows the $\mathrm{rms}$ rotations of the 56 quadrupoles, the $48 \mathrm{BPMs}$, and the 90 steering magnets. Also shown are the maximum rotations and the resolution with which we could determine the rotations. The resolutions come from table 1 .

\begin{tabular}{|c|c|c|c|}
\hline FIT ROTATIONS & rms & maximum & resolution \\
\hline quadrupole & $1.4 \mathrm{mrad}$ & $3.6 \mathrm{mrad}$ & $.4 \mathrm{mrad}$ \\
BPM & $10 \mathrm{mrad}$ & $31 \mathrm{mrad}$ & $.5 \mathrm{mrad}$ \\
steering magnet & $6 \mathrm{mrad}$ & $21 \mathrm{mrad}$ & $.8 \mathrm{mrad}$ \\
\hline
\end{tabular}

The first work that was a direct application of the better understanding of the X-Ray Ring optics was the development of a low emittance lattice [12]. The response matrix fit was done using response matrices measured while the sextupole magnets were turned off. We then turned on the sextupoles and remeasured the response matrix. Starting with the MAD model which was generated by fitting the response matrix with the sextupoles off, we varied gradients in each sextupole to fit the matrix measured with the sextupoles on. In this way we were able to derive the gradients in each of the sextupoles due to horizontal orbit offsets in the sextupoles. We then adjusted the strengths of the quadrupoles adjacent to the sextupoles in order to compensate for the sextupole gradients. Thus we were able to correct a large break in periodicity of the dispersion, and reduce the horizontal emittance.

Another application of the results will be in the X-Ray Ring coupling correction algorithm [13]. The coupling algorithm is limited by previously unknown corrector and BPM rotational misalignments. Now that these rotations are known, they will be used to improve the coupling correction.

\section{CONCLUSION}

Analysis of the measured orbit response matrix has yielded a great deal of detailed information concerning the $\mathrm{X}$-Ray Ring including the normal and skew gradients in each quadrupole. This information has already proven useful for lowering the horizontal emittance. The BPM, steering magnet, and quadrupole rotation information should improve the X-Ray Ring coupling correction. The results from this analysis would be useful for colliding beam storage rings and damping rings as well as synchrotron light sources.

\section{ACKNOWLEDGEMENTS}

I would like to thank Jeff Corbett, Sam Krinsky, and Martin Lee for stimulating discussions. Susila Ramamoorthy's improved fast averaged orbit readings helped provide the accurate orbit measurements necessary for this work. John Smith's help providing cpu time for the lengthy calculations was appreciated, as was Yong Tang's help integrating the program with the NSLS control system. Thanks to Julie Leader for her patient editing.

\section{References}

[1] N.M. Gelfand, Coupling in the Tevatron, FERMILABTM-1916, Dec, 1994.

[2] J. Safranek and M.J. Lee, Calibration of the X-Ray Ring Quadrupoles, BPMs, and Orbit Correctors Using the Measured Orbit Response Matrix, AIP Conference Proceedings, Vol 315, 1994.

[3] J. Safranek and M.J. Lee, Proceedings of the $1994 \mathrm{Eu}$ ropean Particle Accelerator Conference, pg 1027.

[4] W.J. Corbett, M.J. Lee and V. Ziemann, "A Fast Model-Calibration Procedure for Storage Rings," SLAC-PUB-6111, May, 1993.

[5] M.J. Lee, Y. Zambre, W.J. Corbett, "Accelerator Simulation Using Computers," SLAC-PUB-5701A, 1991.

[6] S. Kamada, Proceedings of the Workshop on Non Linear Dynamics in Particle Accelerators, 1994.

[7] J. Bengtsson and M. Meddahi, Modeling of Beam Dynamics and Comparison with Measurements for the Advanced Light Source, Proceedings of the 1994 European Particle Accelerator Conference, pg 1021.

[8] R. Biscardi and J.W. Bittner,Switched Detector for Beam Position Monitor, Proceedings of the 1989 IEEE Particle Acclerator Conference, pg 1516.

[9] H. Grote,F.C. Iselin, The MAD Program, Version 8.1, CERN/SL/90-13, June 17, 1991.

[10] W. Press, B. Flannery, S. Teukolsky, W. Vetterling, Numerical Recipes, Cambridge, 1990.

[11] R. Nawrocky, to be published.

[12] J. Safranek, A Low Emittance Lattice for the X-Ray Ring, these proceedings.

[13] J. Safranek and S. Krinsky, Plans to Increase Source Brightness of NSLS X-Ray Ring, Proceedings of the 1993 Particle Accelerator Conference, pg 1491. 